\title{
Clinical effects of selenium yeast and levothyroxine combined therapy on patients with lymphocytic thyroiditis.
}

\author{
Di Wu*, Li Jin, Hongshuang Xu
}

Second Department of Endocrinology, Third Affiliated Hospital of Qiqihar Medical School, Qiqihar, Heilongjiang, PR China

\begin{abstract}
Objective: This paper aims to discuss the clinical effects of selenium yeast and levothyroxine combined therapy on patients with lymphocytic thyroiditis.

Methods: Eighty patients with lymphocytic thyroiditis, who were admitted in our hospital from July 2015 to June 2017, were enrolled and randomly and equally divided into control and experimental groups. The control group was treated with levothyroxine, whereas the experimental group was treated with selenium yeast and levothyroxine combined therapy. The clinical effects and relevant indexes of the two groups were statistically analysed.

Results: The experimental group presented a total therapeutic effect of $90.0 \%$, which was considerably higher than that of the control group $(\mathbf{7 0 . 0 \%})$, and the difference was statistically significant $(P<0.05)$. The thyroid stimulating hormone, thyroid peroxidase antibody, and thyroglobulin antibodies of the experimental group were significantly lower than those of the control group $(\mathbf{P}<0.05)$. The peripheral blood T-lymphocyte subpopulation indexes (i.e., CD4/CD3, CD4/CD8, and CD8/CD3) of the experimental group were significantly higher than those of the control group $(\mathbf{P}<0.05)$. The recovery time and total treatment time of the experimental group were substantially shorter than those of the control group $(\mathbf{P}<\mathbf{0 . 0 5})$.

Conclusions: Selenium yeast and levothyroxine combined therapy demonstrated outstanding clinical effects on patients with lymphocytic thyroiditis, effectively improving the thyroid function and accelerating the recovery of the patients. Therefore, this combined therapy strategy has promising potential for clinical application.
\end{abstract}

Keywords: Lymphocytic thyroiditis, Levothyroxine, Clinical effect.

Accepted on October 25, 2017

\section{Introduction}

Lymphocytic thyroiditis is a chronic autoimmune disease that uses thyroid glands as antigens. Hypothyroidism is the clinical manifestation of lymphocytic thyroiditis, whose incidence and development are reportedly related to inheritance, age, psychosis, hormone level, and environmental factors [1,2]. Previous studies have demonstrated that it is mainly caused by $\mathrm{T}$ lymphocyte-mediated cellular immune dysfunction. At present, levothyroxine is widely used in clinical therapy to maintain the thyroid functions of patients. However, this drug exhibits poor clinical effects [3]. Lymphocytic thyroiditis is typically accompanied with abnormal selenium contents and selenoprotein levels, indicating that selenium might be a major cause of lymphocytic thyroiditis. Eighty patients with lymphocytic thyroiditis, who admitted in our hospital from July 2015 to June 2017, were enrolled and divided into two groups. One group was treated with selenium yeast; while the other, with levothyroxine combined therapy. The two groups were compared to explore the clinical effects of the treatments. The results are presented in the subsequent text.

\section{Information and Methods}

\section{General information}

Eighty cases of patients with lymphocytic thyroiditis, who were admitted in our hospital from July 2015 to June 2017, were collected and randomly and equally divided into control and experimental groups. The control group comprised 19 females and 21 males, whose age range was 20-71 y (average: $38.7 \pm 8.7 \mathrm{y}$ ). The shortest course of disease was 1 year, and the longest was $18 \mathrm{y}$ (average: $10.0 \pm 1.5 \mathrm{y}$ ). The experimental group included 22 females and 18 males, whose age range was 21-71 y (average: $38.5 \pm 8.8 \mathrm{y}$ ). The shortest course of disease was $1 \mathrm{y}$, and the longest was $17 \mathrm{y}$ (average: $10.1 \pm 1.4$ ). The two groups were not statistically significant different in terms of gender, age and, course of disease $(\mathrm{P}>0.05)$, indicating that the two groups were comparable. All respondents were informed by the goal of this study and participated voluntarily. All participants signed an Informed Consent form. This study satisfies all ethics requirements. 


\section{Methods}

The control group was subjected to oral intake of levothyroxine (Sichuan Hairong Pharmaceutical Co., Ltd. of Yangzijiang Pharmaceutical Group, SFDA approval number: H20041605) once a day for 2 months at a dosage of $2 \mu \mathrm{g} /$ $(\mathrm{kg} \bullet \mathrm{d})$. The experimental group was treated with selenium yeast (Mudanjiang Ling Tai Pharmaceutical Co. Ltd., SFDA approval number: H10940160) and levothyroxine combined therapy for 2 months. The patients orally ingested selenium yeast twice a day at a dosage of $100 \mu \mathrm{g} /$ time. The application and usage of levothyroxine were the same as those for the control group.

\section{Observation indexes}

The clinical effects and relevant indexes of the two groups were statistically compared. The thyroid functional indexes, namely, Thyroid Stimulating Hormone (TSH), Thyroid Peroxidase Antibody (TPOAb), and Thyroglobulin Antibodies (TGAb), of the two groups were statistically compared. The peripheral blood T-lymphocyte subpopulation indexes, namely, CD4/CD3, CD4/CD8, and CD8/CD3, of the two groups were also compared. The recovery and total treatment times of the two groups were compared as well.

\section{Evaluation standards of clinical effects}

Significantly effective: TPOAb and TGAb decreased significantly $(\geq 50 \%)$ relative to the basic value after treatment. Effective: TPOAb and TGAb are decreased $(<50 \%)$ after treatment. Ineffective: The aforementioned standards were not satisfied after treatment. Total therapeutic effect $=$ (significantly effective cases + effective cases)/total cases $\times 100 \%$.

\section{Statistical analysis}

The clinical data of the two groups were inputted into SPSS 20.0 for analysis. The enumeration data (total therapeutic effect) were expressed as percentages and verified by $\chi^{2}$-test. The measurement data (i.e., TSH, TPOAb, TGAb, CD4/CD3, $\mathrm{CD} 4 / \mathrm{CD} 8, \mathrm{CD} 8 / \mathrm{CD} 3$, recovery time, and total treatment time) were expressed in $(\overline{\mathrm{x}} \pm \mathrm{S})$ and verified by t-test. $\mathrm{P}<0.05$ indicated a statistically significant difference between the two groups.

\section{Results}

\section{Comparison of clinical effects}

The total therapeutic effect of the experimental group was $90.0 \%$, which was significantly higher than that of the control group $(70.0 \%)$, and the difference was statistically significant $(\mathrm{P}<0.05$; Table 1$)$.

\section{Comparison of TSH, TPOAb, and TGAb}

The TSH, TPOAb, and TGAb of the experimental group were significantly lower than those of the control group $(\mathrm{P}<0.05$; Table 2).

\section{Comparison of peripheral blood T-lymphocyte subpopulation indexes}

The peripheral blood T-lymphocyte subpopulation indexes (i.e., CD4/CD3, CD4/CD8, and CD8/CD3) of the experimental group were significantly higher than those of the control group $(\mathrm{P}<0.05)$ (Table 3).

\section{Comparison of recovery time and total treatment time}

The recovery and total treatment times of the experimental group were considerably shorter than those of the control group $(\mathrm{P}<0.05$; Table 4$)$.

Table 1. Comparison of clinical effects between the two groups (n (\%)).

\begin{tabular}{lllll}
\hline Groups & $\begin{array}{l}\text { Significantly } \\
\text { effective }\end{array}$ & Effective & Ineffective & $\begin{array}{l}\text { Total } \\
\text { therapeutic } \\
\text { effect }\end{array}$ \\
\hline $\begin{array}{l}\text { Experimental } \\
\text { group }(n=40)\end{array}$ & $25(62.5)$ & $11(27.5)$ & $4(10.0)$ & $36(90.0)$ \\
\hline $\begin{array}{l}\text { Control } \\
\text { group }(n=40)\end{array}$ & $18(45.0)$ & $10(25.0)$ & $12(30.0)$ & $28(70.0)$ \\
\hline$X^{2}$ & & & 5.0000 \\
\hline$P$ & & & 0.0253 \\
\hline
\end{tabular}

Table 2. Comparison of TSH, TPOAb, and TGAb between the two groups $(\bar{x} \pm S)$.

\begin{tabular}{llll}
\hline Groups & TSH $(\mathrm{mU} / \mathrm{L})$ & TPOAb $(\mathrm{U} / \mathrm{ml})$ & TGAb $(\mathrm{U} / \mathrm{ml})$ \\
\hline Experimental group $(\mathrm{n}=40)$ & $4.8 \pm 1.0$ & $122.4 \pm 18.9$ & $67.9 \pm 9.2$ \\
\hline Control group $(\mathrm{n}=40)$ & $8.0 \pm 1.4$ & $217.3 \pm 25.5$ & $89.0 \pm 10.5$ \\
\hline $\mathrm{t}$ & 11.7634 & 18.9095 & 9.5591 \\
\hline $\mathrm{P}$ & 0.0000 & 0.0000 & 0.0000 \\
\hline
\end{tabular}

Table 3. Comparison of peripheral blood T-lymphocyte subpopulation indexes between the two groups $(\bar{x} \pm S)$.

\begin{tabular}{llll}
\hline Groups & CD4/CD3 & CD4/CD8 & CD8/CD3 \\
\hline Experimental group $(n=40)$ & $44.2 \pm 5.5$ & $1.9 \pm 0.5$ & $27.2 \pm 3.1$ \\
\hline Control group $(n=40)$ & $31.0 \pm 4.7$ & $1.2 \pm 0.3$ & $24.2 \pm 2.6$ \\
\hline$t$ & 11.5395 & 7.5925 & 4.6895 \\
\hline$P$ & 0.0000 & 0.0000 & 0.0000 \\
\hline
\end{tabular}

Table 4. Comparison of recovery time and total treatment time between the two groups $(\bar{x} \pm$ s.d).

\begin{tabular}{lll}
\hline Groups & Recovery time & Total treatment time \\
\hline Experimental group $(n=40)$ & $20.0 \pm 3.1$ & $28.3 \pm 4.4$ \\
\hline Control group $(n=40)$ & $25.7 \pm 3.6$ & $33.9 \pm 4.7$ \\
\hline $\mathrm{t}$ & 7.5882 & 5.5011 \\
\hline
\end{tabular}




\begin{tabular}{lll}
\hline$P$ & 0.0000 & 0.0000 \\
\hline
\end{tabular}

\section{Discussions}

Lymphocytic thyroiditis is a common thyroiditis in clinics. This disease presents no evident clinical manifestations and develops slowly. It has an incubation period of 2-4 years before an obvious goiter is detected. The clinical symptoms of lymphocytic thyroiditis include thyroid diffusion, bilateral symmetry, and malaise, all of which can easily cause hypothyroidism among patients [4]. The annual morbidity of lymphocytic thyroiditis has been steadily increasing in recent years and is becoming close to the incidence of hyperthyroidism. At present, no unified clinical diagnosis standard has been established for lymphocytic thyroiditis. In general, it can be diagnosed based on the following indexes and indicators: sharp growth in blood TSH; positive tests of $\mathrm{TPOAb}$ and TGAb; irregular concentration or sparsity of thyroid glands in scanning images; tough texture and diffusion of thyroid glands; and positive potassium perchlorate excretion test $[5,6]$. The pathological features of lymphocytic thyroiditis include the large-scale infiltration of lymphocytes into thyroid glands and the sharp growth in serum thyroid autoantibody levels. Thus, clinical therapy must follow a set of strict rules, which should include adjusting the body immunity, correcting the cellular immune dysfunction, and maintaining normal secretion of the thyroid glands [7].

Previous studies have reported that lymphocytic thyroiditis is related to abnormal selenium and selenoprotein contents. Selenium affects the TPOAb level and immune modulating functions. Reduction of selenium content causes changes in the T-lymphocyte subpopulation, leading to imbalance [8]. A timely supply of selenium can adjust TPOAb and TGAb, thereby reducing the infiltration of lymphocytes.

Selenium is an important microelement that maintains normal metabolism of the body. Selenium content directly influences the life activities of mammalians. In human bodies, selenium mainly exists as selenoprotein, which is involved in immunoregulation, oxidation resistance, and anti-inflammatory activities. Moreover, selenium not only participates in the secretion and synthesis of thyroid hormones, but also enhances the bioactivity of peroxidase, thus accelerating the scavenging of free radicals [9]. Other roles played by selenium include activating immunity, significantly improving the proliferation and differentiation of B-lymphocyte activity, as well as adjusting T-lymphocyte immune functions and cytokines secretion to enhance $\mathrm{NK}$ and Tc cellular immune killing activity. A shortage of selenium decreases the activities of thioredoxin reductase and glutathion peroxidase [10]. In turn, these reduced activities will decrease the oxidation resistance of the body, accumulate abundant thyroid oxygen free radicals, destroy thyroid cells, influence thyroid endocrine functions, form a vicious circle, and induce an autoimmune thyroid. Therefore, selenium yeast should be taken orally without levothyroxine to weaken thyroid protein levels and protect normal thyroid glands. The results of this study revealed that the experimental group was significantly superior to the control group in terms of total therapeutic effect, TSH, TPOAb, TGAb, CD4/CD3, CD4/CD8, CD8/CD3, recovery time, and total treatment time $(\mathrm{P}<0.05)$. These findings are consistent with the literature, suggesting that selenium yeast and levothyroxine combined therapy achieves better clinical effects than levothyroxine therapy.

\section{Conclusion}

Selenium yeast and levothyroxine combined therapy presented significantly better clinical effects on patients with lymphocytic thyroiditis. This combined treatment effectively improves thyroid function and accelerates recovery, demonstrating its promising potential for clinical application.

\section{References}

1. Loh KC, Greenspan FS, Dong F, Miller TR, Yeo PP. Influence of lymphocytic thyroiditis on the prognostic outcome of patients with papillary thyroid carcinoma. J Clin Endocr Metab 1999; 84: 458-463.

2. $\mathrm{Pu} \mathrm{J}, \mathrm{Xu}$ YY, Dai TT, Peng J. Exposure of acetochlor regulates the activity of udp-glucuronosyltransferases (UGTs) involved in the metabolic elimination of esophageal cancer treatment drugs. Lat Am J Pharm 2017; 36: 1597-1601.

3. Luo J, Zhao T, Liang C. The impact of trimetazidine addition to conservative treatment on hs-CRP, NT-pro-BNP, hs-cTnT and plasma lipid levels in patients with non-ST segment elevation acute coronary syndrome. Biomed Res India 2017; 28: 6225-6229.

4. Allen EM, Rajatanavin R, Nogimori T, Cushing G, Ingbar $\mathrm{SH}$, Braverman LE. The effect of methimazole on the development of spontaneous lymphocytic thyroiditis in the diabetes-prone BB/W rat. Am J Med Sci 1986; 292: 267-271.

5. Okayasu I, Fujiwara M, Hara Y, Tanaka Y, Rose NR. Association of chronic lymphocytic thyroiditis and thyroid papillary carcinoma. A study of surgical cases among Japanese, and white and African Americans. Cancer 1995; 76: 2312-2318.

6. Al-Harbi SA. Novel metal complexes based anti-cancer and anti-microbial agents: synthesis, characterization, molecular docking and pharmacological activities. Lat Am J Pharm 2017; 36: 1429-1443.

7. Marcocci C, Vitti P, Cetani F, Catalano F, Concetti R, Pinchera A. Thyroid ultrasonography helps to identify patients with diffuse lymphocytic thyroiditis who are prone to develop hypothyroidism. J Clin Endocrinol Metab 1991; 72: 209-213.

8. Forati S, Yaghmaii B, Allah-Verdi B. The effect of early feeding after enema reduction of intussusception in order to investigate the rate of recurrence and side effects of reduction. Biomed Res India 2017; 28: 5642-5645.

9. Nikolai TF, Turney SL, Roberts RC. Postpartum lymphocytic thyroiditis. Prevalence, clinical course, and long-term follow-up. Arch Intern Med 1987; 147: 221-224. 
10. Ghorab MM, Alsaid MS, Alqasoumi SI, Abdel-Kader MS. Semisynthesis of some novel urea, thiourea, carbamimidothioic acid and dihydrooxazole derivatives as a new class of anticancer agents. Lat Am J Pharm 2017; 36: 380-385.

\section{*Correspondence to}

Di Wu

Second Department of Endocrinology

Third Affiliated Hospital of Qiqihar Medical School

PR China 